\title{
Index for Volume 22 of Molecular Plant-Microbe Interactions
}

\section{AUTHOR AND SUBJECT INDEX}

Abbà, S., 1412

Accotto, G. P, 1504

Acidovorax avenae, on cucurbits, type IV pili requirement, 909

Adhikari, T. B., 1056

Adrian, M., 977

Agrobacterium spp.

-A. radiobacter, crown gall biocontrol, gene requirements, 713

-A. tumefaciens, Agrobacterium radiobacter for control, gene requirements, 713; lactonases role, regulation, 529; T-DNA transfer, type IV secretion system signaling, 1356

Alfano, J. R., 1069, 1479

Alfenas-Zerbini, P., 352

Ali, S., 1056

Alkan, N., 1484

Allègre, M., 977

Allen, C., 773

Almeida, N. F., 52

Alternaria spp.

-A. alternata, on citrus, oxidative stress tolerance, 942

-A. brassicicola, depudecin, biosynthesis and virulence, 1258

Alvarez, B., 713

Alves de Melo, S., 39

Ambrosino, P., 1021

Ameline-Torregrosa, C., 1043

Ammar, E.-D., 18

Amodeo, G., 1169

Anca, I.-A., 302

Andrés, F., 201

Andrés, M. F., 1081

Andrews, D. L., 1293

Angot, A., 52

Ankala, A., 1555

Aphanomyces euteiches, on Medicago truncatula

—proteasome-related genes, 1043

- protein pattern, proteome, 421

Arabidopsis

- defense responses, constitutive activation, 1635

—downy mildew, host genes, 1104

- patatin-like protein 2 , cell death role, 469

- Pseudomonas syringae, type III effector, plant immunity, 1069

Arabidopsis thaliana: anthracnose, Agrobacterium tumefaciens-mediated transformation, 143; pathosystem, cover photo, July; Pseudomonas syringae, resistance and water loss, 857; Pseudomonas syringae, temperature effect on resistance, 498; resistance, cell-wall mediated, kinase, 953; $R$-like gene regulated, 840; RTM resistance, coat protein, 1302

Arachis hypogea

—endosymbionts dissemination, RNA interference, 1466

-root nodule symbiosis, 132

Arasteh, J. M., 391

Archer, D. B., 1412

Arens, M., 630

Arias, C. M., 341

Arnould, C., 341

Aroca, R., 1169

Arrese-Igor, C., 1565

Asai, S., 619

Aspergillus spp., on maize, lipoxygenase, susceptibility, 222

-A. flavus, host interaction, oxygenase coordination, 882

Astegno, A., 1577

Atsumi, G., 166

Austin-Phillips, S., 437

Avis, T. J., 1479

Azaiez, A., 190

Bacillus subtillis, surfactin-type lipopeptide, defense-related event, 456

Bago, A., 1169

Bahar, O., 909

Bai, J., 1056

Bai, X., 18

Baier, M. C., 63

Bakker, J., 330

Balagué, C., 469

Balesdent, M. H., 725, 932

Balmuth, A. L., 391

Bamboo mosaic virus, cell-to-cell and systemic movement, cysteine residues, 1379

Banasiak, J., 921

Bao, Z., 498

Baranger, A., 1043

Barash, I., 849

Barberis, P., 538

Barley

- penetration resistance and mutase, 311

-powdery mildew, pH signaling, 1179

Barret, M., 1611

Bartetzko, V., 655

Barthélemy, J. P., 456

Basnayake, B. M. V. S., 1227

Beattie, G. A., 857

Becker, A., 1656

Beet necrotic yellow vein virus, on sugar beet, resistance proteins, 999

Bélanger, R. R., 1323
Bella, P., 1514

Bellogín, R. A., 1445

Belzile, F., 1323

Ben, C., 1645

Benhassou, H. A., 341

Berberich, T., 31

Berrocal, M., 953

Bertani, I., 1514

Besnard, A.-L., 932

Betran, J., 222

Beutner, C., 421

Bhaheetharan, J., 882

Bhaskar, P. B., 447

Bianciotto, V., 302

Biological control

- crown gall and Agrobacterium radiobacter, gene requirements, 713

-Trichoderma atroviride, gene and transporter membrane pump, 291

- Trichoderma harzianum, endopolygalacturonase requirement, 1021

Biotroph, root biotroph interaction, jasmonic acid and salicylic acid signaling, review, 763

Birch, P. R. J., 630

Birker, D., 157

Blaise, F., 725

Blein, J.-P., 868

Blumeria graminis

—on barley, penetration resistance, 311

-host epicenter wax, lipase, 1601

Bolton, M. D., 487

Bonfante, P., 302

Börnke, F., 655

Bos, J. I. B., 269

Botryis cinerea

- on Nicotiana benthamiana, resistance and nitric oxide, 619

-on strawberry and grape, activator protein 1, oxidative stress, 987

-on tomato, hexanoic acid-induced resistance, 1455

Bottin, A., 1043

Boucher, C., 538

Boutin, M., 1611

Bouwmeester, K., 1535

Boyle, B., 190

Bradeen, J. M., 362, 437

Brassica napus

—Leptosphaeria maculans, pathogenicity determinants, 725

- Pseudomonas putida, bacterial proteins and interaction, 686

-Sclerotinia sclerotiorum: jasmonic acid-mediated defense, 235; virulence and gene disruption of precursor, 783 
Bremia lactucae, on lettuce, quantitative trait loci, 1160

Brendel, M., 39

Brenkman, A. B., 1250

Brigneti, G., 589

Brodhagen, M., 222

Brommonschenkel, S. H., 352

Brown, S. H., 222

Brun, H., 932

Brunner, F., 790

Bücking, H., 1492

Buendía-Clavería, A. M., 575

Burdman, S., 909

Bureau, C., 747

Cabauatan, P. Q., 1268

Cabunagan, R. C., 1268

Cai, L., 235

Cailleteau, B., 868

'Candidatus Glomeribacter

gigasporarum', symbiotic phases, strigolactone, 302

'Candidatus Liberibacter asiaticus'

-on citrus, diversity, metagenomic approach, 1624

-genome sequence of bacteria, metagenomics, 1011

'Candidatus' Phytoplasma asteris, plant cell nuclei targeted by protein, 18

Candresse, T., 1302

Cano, C., 1169

Canola (see Brassica napus)

Canto, T., 642

Capsicum annuum, oxygen and nitric oxide, cell death, 1389

Cardoza, R. E., 1021

Carputo, D., 362, 437

Carr, J. P., 642

Cascardo, J. C. M., 352

Catara, V., 1514

Catoni, M., 1504

Cazale-Noel, A.-C., 538

Cazaux, M., 1043

Cen, W.-J., 1401

Cesbron, S., 747

Chain, F., 1323

Chakrabarti, A., 1191, 1214

Chalupowicz, L., 849

Champouret, N., 1535

Chang, B.-Y., 1379

Chaparro-Garcia, A., 269

Chardon, F., 1043

Chattoo, B. B., 1635

Chaudhury, S. R., 132

Chen, S., 1128

Cheng, C.-J., 1143

Cheng, Z., 686

Chestnut, blight, silencing and virulence, 211

Cheung, K. W. J.-T., 1546

Cho, S., 1366

Choi, H. W., 1389

Choi, I.-R., 1268

Choi, S. H., 642

Chou, Y.-L., 1379

Choudoir, M. J., 447

Chronis, D., 1128
Chu, A. L., 665

Chung, K.-R., 942

Cillo, F., 1239

Cirou, A., 529

Citrus

-brown spot, oxidative stress tolerance, 942

'Candidatus Liberibacter asiaticus', diversity, metagenomic approach, 1624

-huanglongbing bacterium, genome sequence, 1011

Ciuffetti, L. M., 665

Cladosporium fulvum, on tomato

-disease resistance protein, leucine, 1203

-interaction specificity, cover photo, October

-leaf mold, cover photo, March

-resistance protein, 1214

-specificity and evolution of pathosystem, review, 1191

-transcriptional responses, 245

Clara da Silva, A., 39

Clavibacter michiganensis, on potato, hypersensitivity, serine protease, 809

Clover yellow vein virus, on pea, salicylic acid signaling, 166

Cochliobolus heterostrostrophus, MAP kinase signaling, phosphorylation, 1093

Colditz, F., 421

Colletotrichum spp.

$-C$. coccodes, on tomato fruits, ammonium and oxidase activity, cell death, 1484

-C. higginisianum: on Arabidopsis thaliana, mutagenesis, cover photo, February; pathogenicity gene, mutagenesis, 143

Collmer, A., 52, 1341

Condon, B., 52

Coplin, D. L., 703

Correa, V. R., 18

Cosson, P., 1302

Coté-Beaulieu, C., 1323

Cotton leaf curl Multan virus, ubiquitinconjugating enzyme requirement, 737

Cover photo

-Cladosporium fulvum, on tomato: interaction specificity, October; leaf mold, March

-Colletotrichum higginisianum, on Arabidopsis thaliana, mutagenesis, February

-Fusarium graminearum on wheat, in phloem, August

-Medicago truncatula, carbon and nitrogen metabolism in root nodules, December

-Peltigera leucophlebia, cyanobacteria in cephalodia, June

-Phymatotrichopsis omnivora, on alfalfa, January

-Phytophthora infestans, on potato, fluorescent signals, April —potato, late blight, leaf symptoms, May

-Pseudomonas syringae and $P$. fluorescens: on Arabidopsis, hypersensitivity, September; Arabidopsis thaliana pathosystem, July; on tomato, bacterial speck, chlorosis, November

Cowan, G. H., 381

Creissen, G., 1635

Crespo-Rivas, J. C., 575

Crimi, M., 1577

Cryphonectria parasitica, on chestnut, silencing and virulence, 211

Cucumber mosaic virus

-on Arabidopsis, patatin-like protein 2, cell death role, 469

- protein $2 \mathrm{~b}$, role in symptoms, 642

-on tomato, strains and RNA 2 sequence, 1239

Cucurbits, bacterial fruit blotch, type IV pili requirement, 909

Cunnac, S., 538

Daire, X., 977

Darrasse, A., 747

Darsonval, A., 747

Das, A., 73

DasGupta, M., 132, 1466

da Silva Gesteira, A., 39

Davydov, O., 1484

Dawson, W. O., 1624

Deák, V., 1422

Deakin, W. J., 519

Decroocq, V., 1302

de Groot, P. J., 245

de Jong, M., 1104

Dekter, R. W., 1104

de la Oleyva, M., 1455

de la Peña, M., 1203

Delibes, A., 1081

Deller, S., 790

de Mattos Cardo, J. C., 39

De Moraes, C. M., 551

del Socorro Murdoch, P., 575

Deng, X., 964

Depudecin, Alternaria brassicicola, virulence role, 1258

Deslandes, L., 538

Dessaint, F., 341

Dessaux, Y., 529

Detector biology plant organisms, concepts, review, 115

de Wit, P. J. G. M., 245

Díaz-Ruíz, J. R., 1431

Dickerman, A., 1011

Di Pietro, A., 830

Dixon, R. A., 7

Djébali, N., 1043

Doddapaneni, H., 1011

Dogra, S., 737

Domingo, C., 201

Dommes, J., 456

Dong, C., 235

Dry, I. B., 737

Duan, J., 686

Duan, Y., 1011 
Duby, F., 456

Dumas, B., 1043

Durand, K., 747

Egan, J. D., 1293

Eini, O., 737

Elarbi Aouani, M., 1043

Elmayan, T., 868

Encabo, J. R., 1268

Ermel, M., 932

Erwinia amylovora, secretion, translocation, and binding, analysis, 1282

Esquerré-Tugayé, M.-T., 1043

Estévez, J. M., 953

Evans, C., 52

Ewald, D., 1032

Fan P.-S., 1143

Fan, Z. F., 1523

Farrand, S. K., 713

Faure, D., 529

Fávaro, R. D., 352

Fehlberg, V., 63

Felle, H. H., 1179

Feng, J., 1601

Feng, J.-X., 321, 1401

Feng, Q., 411

Feussner, I., 222, 469

Fewer, D. P., 695

Feys, B. J., 1645

Figlerowicz, M., 921

Finan, T. M., 1116

Finiti, I., 1455

Fiorilli, V., 1504

Flemetakis, E., 800

Flores-Cruz, Z., 773

Flors, V., 1455

Fluhr, R., 1484

Foster, S. J., 589, 601

Fowler, J. E., 1116

Fradin, E. F., 245

Freeman, B. C., 857

Frey-Klett, P., 1611

Fromentin, J., 868

Fu, D.-Q., 86

Fu, H., 235

Fudal, I., 932

Fungicides, carbendazim, cereal head blight resistance, 1143

Furutani, A., 96

Fusarium spp.

-F. graminearum: on cereal, carbendazim resistance, trichothecene, 1143; deoxynivalenol genes, 1588; on wheat, phloem, cover photo, August; on wheat, transcriptome analysis, 1366; on wheat, triacylglyceride metabolism, 1492; on wheat, tricothecene pathway, 899

$-F$. oxysporum on tomato: root infection, CWDE gene expression, 507; virulence and transcription factors, 830

Gabriel, D. W., 1011
Gaeumannomyces graminis, on wheat, biocontrol, genes, 1611

Gallitelli, D., 1239

Gao, X., 222

García, J. A., 1302

García-Agustín, P., 1455

García-Marco, A., 1431

García-Pedrajas, M. D., 1293

Ge, Y.-Y., 1401

Gene, aquaporin, Glomus intradices, expression analysis, 1169

Genetics, gene-for-gene interactions, oomycete effectors, review, 371

Genin, S., 538

Gentzbittel, L., 1645

Geoffroy, P., 469

Ghabrial, S., 86

Ghignone, S., 302

Gianinazzi-Pearson, V., 341

Gibon, Y., 800

Gijzen, M., 1479

Gil-Serrano, A., 575

Glasa, M., 1302

Glick, B. R., 686

Globodera rostochiensis

—on potato, CLE-like genes, 1128

-on tomato, SPRY domain-containing protein, 330

Glomus intradices

-on aquaporin and gene expression, 116

-on Medicago truncatula, symbiosisrelated plant genes, 341

Gnorimoschema gallaesolidaginis, on Solidago altissina, gall and phytohormones, 551

Göbel, C., 222, 469

Godfroy, O., 341

Goffer, T., 909

Gold, S. E., 1293

Goldbach, R., 1250

González, E. M., 1565

González-Bosch, C., 1455

González-Jara, P., 1431

Gottwald, T., 1011

Gouzy, J., 341

Govers, F., 1535

Goverse, A., 330

Gowda, S., 1624

Gramacho, K. P., 39

Grape, downy mildew, stomata and elicitor-induced protection, 977

Greenshields, D., 1601

Guasch-Vidal, B., 1445

Gudmestad, N. C., 1056

Guenther, J. C., 1492

Guernec, G., 1611

Guillerm-Erckelboudt, A.-Y., 1611

Günther, C., 800

Guo, M., 1069

Gurung, S., 1056

Gutiérrz, S., 1021

Gutjahr, C., 763

Guttman, D. S., 176

Haapalainen, M., 282

Hadeler, B., 899

Hall, D. G., 1011
Hallen-Adams, H. E., 1492

Ham, J. H., 703

Hammes, U. Z., 655

Hammond-Kosack, K. E., 560, 790, 1479

Han, B., 411

Hang, X.-H., 1401

Hao, Y., 686

Hardiner, D. M., 1588

Harrison, K., 1214

Hartner, K., 655

Hase, Y., 259

Hasegawa, M., 820

Hashimoto, M., 677

Hassani-Mehraban, A., 1250

Haudecoeur, E., 529

Hause, B., 63

He, C., 402

He, S.-Y., 703

He, Y.-Q., 321, 1401

Heese, A., 1203

Heitz, T., 469

Héloir, M.-C., 977

Henry, G., 456

Hermosa, R., 1021

Hernández-Blanco, C., 953

Heterodera avenae, on wheat, class III peroxidase genes, 1081

Heupink, A., 1535

Hidalgo, A., 575

Hill, J. H., 123

Himeno, M., 677

Hodge, S., 1645

Hogenhout, S. A., 18, 115

Hohnjec, N., 63

Hollomon, D. W., 1143

Hooykaas, P. J. J., 1356

Hori, H., 1331

Horowitz Brown, S., 882

Horwitz, B. A., 1093

Houjyou, Y., 1331

Hsu, H.-T., 1379

Hsu, Y.-H., 1379

Hu, C.-C., 1379

$\mathrm{Hu}, \mathrm{P} ., 311$

$\mathrm{Hu}$, Y., 411

Hua, J., 498, 840

Huang, D.-L., 321

Huang, J.-D., 1401

Hughes, G. R., 1601

Huguet, T., 1043, 1645

Huibers, R. P., 1104

Huser, A., 143

Hwang, B. K., 1389

Hyaloperonospora arabidopsidis, on Arabidopsis, host genes, 1104

Hypersensitivity

-Clavibacter michiganensis on potato, 809

- pea aphid, race-specific resistance on Medicago, 1645

- Phytophthora infestans, effector R3a, cell death, 269

-Soybean mosaic virus, inclusion protein, 1151

-type III detector proteins, on tomato and Arabidopsis, bacteria, 703 
-Xanthomonas campestris, Zur, hrp cluster, 321

Iannino, F., 519

Iglesias, D. J., 201

Ilgen, P., 899

Inoue, H., 820

Instructions for authors, 107

Iorizzo, M., 362, 437

Isakeit, T., 222

Ishikawa, A., 1331

Ishimaru, C. A., 809

Ismail, N., 1645

Jacobsen, E., 601, 630, 1535

Jacob-Wilk, D., 211

Jacques, M.-A., 747

Jacquet, C., 1043

Jagger, L., 589

James, E. K., 800

Jasinski, M., 921

Jaueau, A., 1043

Jaulneau, V., 1043

Jeuken, M. J. W., 1160

Ji, R., 235

Jia, H., 1366

Jiang, B.-L., 321, 1401

Jiang, C.-J., 820

Jiang, G.-F., 1401

Jiang, J., 437, 447

Jiang, W., 1401

Jofré, F., 1656

Jokela, J., 695

Jones, D. A., 1191, 1203, 1214

Jones, J. D., 52

Jones, J. D. G., 157, 589, 601, 1203, 1214

Jonkers, W., 507

Jordá, L., 953

Jourdan, E., 456

Kaasalainen, U., 695

Kachroo, A., 86

Kagaya, U., 166

Kagiwada, S., 677

Kalitkiewicz, A., 921

Kaminskyj, S., 1601

Kamoun, S., 18, 115, 269

Kanyuka, K., 560

Kanzaki, H., 31

Kav, N. N. V., 783

Kawaguchi, M., 259

Kawanishi, T., 677

Kazan, K., 1588

Kazmierczak, P., 211

Kekker, N. P., 882

Keller, N. P., 222

Kemen, E., 52

Kempa, S., 1565

Kerepesi, I., 1422

Khouja, H. R., 1412

Kikuchi, S., 1268

Kim, K.-H., 1151, 1258

Kitazawa, H., 166

Knudson, D. L., 809

Knudson, S. E., 809

Kodaira, T., 1331
Kogel, K.-H., 1179

Kolomiets, M. V., 222

Komatsu, K., 677

Komatsu, T., 1331

Kondon, H., 1268

Kormelink., R., 1250

Kouchi, H., 259

Kramer, L. C., 437, 447

Kravchuk, Z., 1455

Küfner, I., 790

Kuhn, D., 725

Kuhn, M.-L., 932

Kusano, T., 31

Küster, H., 63

Kutluk Yilmaz, N. D., 560

Kvitko, B. H., 1341

Kwak, D.-Y., 1268

La Camera, S., 469

Lactuca saligna, downy mildew, resistance and quantitative trait loci, 1160

Ladera, R., 1565

Lamb, C., 1635

Lan, L., 964

Lanfranco, L., 1504

Lanzuise, S., 291

Larrainzar, E., 1565

Lawrence, C. B., 1258

Lee, D. H., 1389

Lee, H.-K., 7

Lee, J.-H., 1268

Lee, S.-H., 1151

Legrand, M., 469

Lenz, F., 63

Lepek, V. C., 519

Leptosphaeria maculans

-on oilseed rape, pathogenicity determinants, enzyme, 725

-virulence evolution, alternative mechanism, 932

Lettuce, downy mildew, quantitative trait loci, 1160

Lev, S., 1093

Levée, V., 190

Lewsey, M., 642

Lherminier, J., 868

Li, Guang-Hua, 1401

Li, Guihua, 402

Li, Guojun, 1227

Li, H. F., 1523

Li, W., 411, 1227

Li, X.-Q., 321

Li, Y., 840

Liang, Y., 783

Liao, Q., 321

Licciardello, G., 1514

Lin, C., 402

Lin, C.-H., 942

Lin, H., 1011

Lin, N.-S., 1379

Lindeberg, M., 52

Lindhout, B. I., 1356

Liu, G., 1601

Liu, H., 52

Liu, J., 1401

Liu, L., 1011
Liu, S., 235

Llorente, F., 953

Lloret, J., 575

Lokossou, A. A., 630

López, M. M., 713

López-Baena, F. J., 575, 1445

Lopez Braña, I., 1081

Lorito, M., 291, 1021

Lotus japonicus

-Mesorhizobium loti, type-three protein secretion, 519

—nodulation, root regulator, 259

-root nodules, leghemoglobins, 800

Lu, G., 411

Lu, G.-T., 321, 1401

Lu, S.-W., 1128

Lukhovitskaya, N. I., 381

Lumini, E., 302

Luthe, D. S., 1555

Lyons, R., 560

Ma, W., 176

Macalalad, R. J. A., 1268

Mackey, D., 703

MacLean, A. M., 1116

MacLean, D., 52

Maeda, K., 1331

Maeda, S., 820

Maejima, K., 677

Magnaporthe spp.

$-M$. grisea, on rice: auxin and defense response, 201; resistance and desaturase gene, 820

-M. oryzae: on Arabidopsis, preinvasion resistance, PEN2mediated, 1331; on rice, resistance gene Piz-t, 411; zinc-finger protein, conidiation and mycelial infection, 402

Magori, S., 259

Maia, I. G., 352

Maier, F. J., 899

Maize

-Aspergillus spp., lipoxygenase and susceptibility, 222

- defense protein Mir1-CP, ethylene and jasmonic acid signaling, 1555

-smut, calcineurin and virulence, 1293

Majerczak, D. R., 703

Maliepaard, C., 1535

Manners, J. M., 1588

Manning, V. A., 665

Mansfield, J. W., 1645

Manulis-Sasson, S., 849

Mao, H., 235

Marco, Y., 953

Marek, S. M., 7

Margaret, I., 575

Marra, R., 291

Martiáñez, J., 1431

Martin, G. B., 52

Martino, E., 1412

Mascia, T., 1239

Mathé, C., 1043

Matsumura, H., 31

Mattinen, L., 809

McConkey, B. J., 686 
McDonald, B. A., 371

McSpadden Gardener, B. B., 269

Mecey, C., 703

Medicago truncatula

-ABC transporters, 921

-Aphanomyces euteiches: protein pattern and defense, 421; resistance, protein proteasome-genes, 1043

-hypersensitivity, pea aphid, 1645

-nitrogen fixation, regulation, carbon metabolism, 1565; carbon and nitrogen metabolism in root nodules, cover photo, December

-Phymatotrichopsis omnivora, chemical pathways, 7

-signal peptide, nitrogen-fixing symbiosomes, 63

-Sinorhizobium meliloti, symbiosis, N5 gene, 1577

Meinhardt, S. W., 1056

Melampsora spp., on poplar, transcriptome profiling, 190

Melillo, M. T., 1081

Melotto, M., 1282

Meng, Y., 311

Mengiste, T., 1227

Menzies, J. G., 1323

Mesorhizobium loti

—mutants, nodulation, 1546

-type-three protein secretion, characterization, 519

Metzler, M., 809

Meyer, M., 725

Micheli, F., 39

Milde, L., 882

Millett, B. P., 362, 437

Miozzi, L., 1504

Mitchum, M. G., 1128

Molina, A., 953

Molitor, A., 1179

Mollov, D. S., 362, 437

Monreal, J. A., 1445

Monte, E., 1021

Morales, J., 630

Morandi, D., 341

Morán-Diez, E., 1021

Morel, J., 868

Morgan, R. L., 176

Mori, M., 820

Mosely, J. A., 575

Motteram, J., 790

Mucyn, T. S., 391

Muehlbauer, G. J., 1366

Munkvold, K. R., 1341

Mycosphaerella graminicola, on wheat,

NPP1 domain-containing protein, 790

Mycotoxin

-deoxynivalenol, Fusarium graminearum on wheat, genes, 1588

- tox-A., Pyrenophora tritici-repentis, host response, 665, 1056

-trichothecene, Fusarium graminearum: in carbendazim resistance, 1143; pathway, wheat, 899

Mysore, K. S., 7

Myrfield, M., 1056
Nagy, E., 1422

Nagy, T., 1422

Nakahara, K. S., 166

Nakashima, J., 7

Namba, S., 677

Nicotiana benthamiana (see also

Tobacco)

-Botryis cinerea, resistance and nitric oxide, 619

-necrosis, tomato relation, 1214

-nonhost resistance, serine palmitoyltransferase, 31

-phytoplasma, protein cell nuclei, 18

-Potato mop-top virus, RNAs and longdistance movement, 381

- Pseudomonas syringae, temperature effect on resistance, 498

-resistance proteins, cell death induction, 157

-type III secretion system, resistance, 176

Niehaus, K., 421

Niks, R. E., 1160

Nissinen, R., 809

Nitrogen fixation

- Medicago truncatula, carbon metabolism, 1565

-symbiosomes, Medicago truncatula signal peptide, 63

Nodulation

-Arachis, endosymbionts dissemination, RNA interference, 1466

-Arachis hypogea, hairy root transformation, 132

-Lotus japonicus: root regulator, 259; symbiotic leghemoglobins, 800

-Mesorhizobium loti, gene mutants, 1546

-Sinorhizobium fredii, mutants, 575

Noguchi, Y., 96

Nomura, K., 703

Nürnberger, T., 790

Ochiai, H., 96

O'Connell, R., 143

Oger, P. M., 713

Oidiodendron maius, conidiation and mycorrhization, gene disruption, 1412

Oka-Kira, E., 259

Oku, T., 96

Ollero, F. J., 575, 1445

Ongena, M., 456

Ott, T., 800

Overmars, H., 330

Ozeki, J., 677

Pacheco, R., 1431

Paenibacillus sp., on poplar, metabolomics, 1032

Pagán, I., 953

Palukaitis, P., 642, 1312

Pálvölgyi, A., 1422

Pandolfini, T., 1577

Panijel, M., 849

Panter, S. N., 1214
Pantoea spp.

-P. agglomerans, on bean, quorumsensing, auxin, Hrp regulon, galls, 849

-P. stewartii, on tomato, virulence factors, 703

Park, T.-H., 589, 601, 610

Pasciuto, M. M., 1239

Paszkowski, U., 763

Patel, J., 1056

Payne, T., 642

Paz, J. A., 1169

Pea, Clover yellow vein virus, salicylic acid signaling, 166

Peeters, N., 538

Pel, M. A., 589, 601

Pelgrom, K., 1160

Peltigera leucophlebi

- cyanobacteria in cephalodia, cover photo, June

-microcystin production, symbiosis, 695

Peng, Y., 1601

Pennington, B. O., 840

Penyalver, R., 713

Pepper yellow mosaic virus, on tomato, genome-wide analysis, 352

Pérez-Montaño, F., 1445

Peroni, E., 1577

Perotto, S., 1412

Petrescu, A.-J., 330

Phaseolus vulgaris, blight, phyllosphere and seed transmission, 747

Phymatotrichopsis omnivora

-on alfalfa, cover photo, January

-on Medicago truncatula, mycelial model, 7

Phytophthora infestans

-hypersensitivity and cell death, effector R3a, 269

-on potato: class I ipiO variants, 1535; leaf symptoms, cover photo, May; resistance genes and linkage group IV, 630; $R$ gene transcript abundance, $362 ; R B$ gene, 447 ; symptoms and fluorescent signals, cover photo, April

-on Solanum venturii, resistance genes, 589,601

Pii, T., 1577

Pilet-Nayel, M.-L., 1043

Pirhonen, M., 282, 809

Piriformospora indica, on barley, 1179

Plant defense

— calmodulin gene, on pepper, 1359

— jasmonic acid and salicylic acid signaling, root biotroph interaction, review, 763

-metabolism, review, 487

Plantago asiatica mosaic virus, cell-tocell movement, coat protein $\mathrm{N}$ terminal, 677

Plasmopara viticola, on grape, elicitorinduced protection, 977

Plastocyanin, Potato virus $X$, coat protein interaction, 1523

Poinsot, V., 1422 
Poplar

-Paenibacillus sp., beneficial interaction, metabolomics, 1032

_rust, transcriptome profiling, 190

Postma, W., 330

Potato (see also Solanum venturi)

-Clavibacter michiganensis, virulence and serine protease, 809

-cyst nematode, CLE-like genes, 1128

-late blight: resistance and $R B$ gene, transcript; $R$ gene variation, phenotypes, 362 abundance, 447; resistance genes, linkage group IV, 630; transcript abundance, 447

-Phytophthora infestans, virulence variants, 1535

-RNA silencing, resistance, 437

Potato mop-top virus, on Nicotiana benthamiana, RNAs and long-

distance movement, 381

Potato virus $X$

-coat protein, plastocyanin, silencing, 1523

-Potato virus $Y$ interaction, transcriptional changes, 1431

Potato virus $Y$, antiviral response genes, 1312

Poueymiro, M., 538

Powell, G., 1645

Powers, S., 560

Prasad. B. D., 1635

Prins, P., 330

Prospéri, J.-M., 1645

Prusky, D., 1484

Pseudomonas spp.

-P. cichorii, on Nicotiana benthamiana, nonhost resistance, sphingolipid biosynthesis, 31

$-P$. corrugata, transcriptional activator rifA, co-transcription, 1514

-P.fluorescens, on Arabidopsis, type III protein secretion system and effector, cover photo, September

-P. putida, on Brassica napus, bacterial proteins, 686

-P. syringae: on Arabidopsis, type III effector, plant immunity, cover photo, September, 1069; on Arabidopsis, virulence factors, 703; on Arabidopsis and Nicotiana spp., temperature effect on resistance, 498; on Arabidopsis thaliana pathosystem, cover photo, July; on Arabidopsis thaliana water loss and vascular activity, 857 ; bean, genemediated and acquired resistance, 86; on tomato, type III effector, chlorosis, 1341; on tomato, type III effector repertoire, genome sequence, 52; type III effector, resistance system, 176; type III secretion system, gene induction, 864; type III secretion system, pilus protein HrpA, 282

Pugin, A., 977

Pungartnik, C., 39
Putnoky, P., 1422

Pyrenophora tritici-repentis

-host responses, tox-A, 1056

-on wheat, host-selective toxin, 665

Qiao, Y., 1523

Qin, L., 330

Quesada-Ocampo, L. M., 269

Raasch, J., 437

Radom, M., 921

Radutoiu, S., 1546

Raffoux, A., 529

Rakhshandehroo, F., 1312

Ralstonia solanacearum

-on tobacco, type III secretion effectors, 538

-on tomato, oxidative environment, 773

Randles, J. W., 737

Rangaraj, N., 73

Rao Uppalapati, S., 7

Rasmussen, J. B., 1056

Rathjen, J. P., 391

Real, M. D., 1455

Reguera, M., 575

Rehman, S., 330

Remy, E., 725

Rep, M., 507

Resistance

-Arabidopsis: to Cucumber mosaic virus, 469; to pathogens, $R$-like gene regulation, 840

-Arabidopsis and Nicotiana spp. to Pseudomonas syringae, temperature effect, 498

-Arabidopsis thaliana to disease, cellwall mediated, 953

-Medicago truncatula to pea aphid, hypersensitivity, 1645

-Nicotiana benthamiana: to Botryis cinerea, nitric oxide, 619 ; to Pseudomonas cichorii, nonhost, 31; proteins and cell death induction, 157

—potato to late blight: $R B$ gene, $447 ; R B$ transgene, 437

- primary metabolism and plant defense, review, 487

-Pseudomonas syringae on soybean and tobacco, type III effector, 176

-rice to blast, preinvasion, 1331; to blast and leaf blight, desaturase gene, 820

-Solanum venturii to late blight, resistance genes, 601

-soybean to: Soybean mosaic virus, inclusion protein, 1151; Soybean mosaic virus and Pseudomonas syringae, 86

- tobacco to pathogens, oxygen species production, 868

-tomato to: Botrytis cinerea, hexanoic acid-induced, 1455; leaf roll, resistance protein, 1214

-wheat to Soilborne cereal mosaic virus, leakiness and coat protein, 560

Revers, F., 1302

Reviewers, acknowledgment of, 3

Rezaian, M. A., 737

Rice

—bacterial blight, adhesion and virulence, 73

-blast: preinvasion resistance, Arabidopsis, 1331; resistance gene Piz-t, protein, 411

-blast and leaf blight, resistance, desaturase gene, 820

-defense responses, constit tive activation, 1635

-Magnoporthe grisea, auxin and defense response, 201

-tungro viruses, suppression by Utri Merah, 1268

Rice tungro spherical virus, tungro virus suppression, 1268

Rietman, H., 601, 1535

Rikkinen, J., 695

Rispail, Y., 830

Robertson, F. C., 642

Roby, D., 469

Rodpothong, P., 1546

Rodrigues, C. D. A., 507

Rodríguez-Carvajal, M. A., 575

Roesch, L. F. W., 1624

Ronson, C. W., 800, 1546

Root, biotroph interaction, jasmonic acid and salicylic acid signaling, review, 763

Rose, M. S., 1093

Ross, S., 932

Rouxel, T., 725, 932

Rudd, J. J., 790

Ruiz-Lozano, J. M., 1169

Ruiz-Sainz, J. E., 575

Ruocco, M., 291

Russell, A. B., 1341

Ruyter-Spira, C., 630

Ryu, K. H., 642

Saccharomyces cerevisiae, on Theobroma cacao, cytotoxicity, copper transport, 39

Sagi, M., 1484

Saha, S., 132

Saitoh, H., 31

Salcedo, C. I., 713

Salvador, B., 1302

Salvioli, A., 302

Sanada, H., 96

Sanchez, C., 519

Sánchez-Rodríguez, C., 953

Sánchez, F., 1479

Sarniguet, A., 1611

Sassa, G., 849

Sato, S., 259

Satoh, K., 1268

Savenkov, E. I., 381

Schäfer, W., 899

Schepetilnikov, M. V., 381

Scherling, C., 1032, 1565

Schmalenbach, W., 143 
Schneider, D. J., 52

Sclerotinia sclerotiorum

-on Brassica napus, jasmonic acidmediated defense, 235

-on canola, gene disruption of precursor, virulence, 783

Scott, J. B., 882

Seddas, P. M. A., 341

Séguin, A., 190

Selth, L. A., 737

Selvaraj, G., 1601

Senshu, H., 677

Seo, J.-K., 1151

Setubal, J. C., 52

Shachar-Hill, Y., 1492

Sharpee, W. C., 882

Shen, W., 1601

Shibata, S., 259

Shimono, M., 820

Sicard, O., 1302

Silicon, wheat transcriptomic analysis, pathogen stress, 1323

Simon, U. K., 725

Simonetti, E., 1081

Simon-Plas, F., 868

Sinharoy, S., 132, 1466

Sinorhizobium spp.

$-S$. fredi: on legumes, mutants and nodulation, 575; Nops secretion, on soybean, 1445

-S. meliloti: legume endosymbiont, hydroxyproline system, 1116; on Medicago truncatula, N5 gene, symbiosis, 1577 ; on polysaccharide synthesis, genetic analysis, 1422; succinoglycan polymer, 1656

Sivonen, K., 695

Sledge, M. K., 7

Śliwka, J., 589

Smant, G., 330

Soilborne cereal mosaic virus, on wheat, resistance and leakiness and coat protein, 560

Solanaceae, Xanthomonas campestris, type III secretion system, cell wall defense response, 655

Solanum venturii (see also Potato)

-late blight, mapping and cloning, resistance genes, 601

—resistance genes, 589

Solidago altissina, galls, phytohormones and host response, 551

Somerville, S., 953

Song, F., 1227

Songsrirote, K., 1546

Sonnewald, S., 655

Sonti, R. V., 73

Soria-Díaz, M. E., 575

Soybean

—nodulation, mutants, 575

-Pseudomonas syringae, type III secretion system, 176

-resistance, $R$ gene and acquired, 86

- silencing vector, viral gene, DNAbased, 123

-Sinorhizobium fredii, Nops secretion, 1445
Soybean mosaic virus

- gene-mediated and acquired resistance, 86

-inclusion protein and hypersensitivity, 1151

Spanu, P., 1479

Spiridon, L.-N., 330

Squires, J., 1312

Stadler, R., 655

Steeves, J. E., 665

Steindler, L., 1514

Stewart, S. A., 1645

Stougaard, J., 1546

Studholme, D. J., 52

Stukenbrock, E. H., 371

Su, L.-L., 1401

$\mathrm{Su}, \mathrm{S} ., 713$

Sugano, S., 820

Sullivan, J. T., 800, 1546

Sumpton, D. P., 575, 1546

Sun, Y., 1011

Sundin, G. W., 1282

Surette, M., 642

Sutka, M., 1169

Svanella-Dumas, L., 1302

Swiderski, M. R., 157

Symbiosis

-Gigaspora margarita and 'Candidatus Glomeribacter gigasporarum', 302

-Peltigera leucophlebia, mycocystin production, 695

Tabata, S., 259

Taira, S., 282

Takahara, H., 143

Takahashi, Y., 31

Takaoka, M., 96

Takatsuji, H., 820

Takeshita, M., 1312

Tal, H., 1093

Talón, M., 201

Tanaka, A., 259

Tang, D.-J., 321, 1401

Tang, J.-L., 321, 1401

Tang, X., 964

Tang, Y., 7

Tannières, M., 529

Tantaoui Elaraqui, K., 868

Temme, N., 987

Tenllado, F., 1431

Terauchi, R., 31, 115

Tharrau, D., 201

Theobroma cacao, Saccharomyces cerevisiae, cytotoxicity modulation, 39

Thiel, H., 999

Thomas-Oates, J. E., 575, 1546

Thomma, B. P. H. J., 245

Thonart, P., 456

Tian, F., 1069

Tobacco (see also Nicotiana benthamiana)

-host-range specificity, Ralstonia solanacearum, 538

-oxygen species, oxidase-mediated, plant defense, 868
Tomato

-bacterial speck and chlorosis, cover photo, November; type III effector, chlorosis, 1341

-Botrytis cinerea, resistance induced by hexanoic acid, 1455

-Cladosporium fulvum, pathosystem specificity, review, 1191

-Colletotrichum coccodes, cell death, ammonium secretion and oxidase activity, 1484

- Cucumber mosaic virus, strains and RNA 2 sequence, 1239

-Fusarium oxysporum, root infection, CWDE genes, 507

-Globodera rostochiensis, SPRY domain-containing protein, 330

-leaf mold, amino acids and leucine, resistance, 1203

-nucleotide-binding domain, Prf, regulation, 391

- potyvirus, gene expression, genomewide analysis, 352

-Pseudomonas syringae, genome sequence, type III effector repertoire, 52

-Pythium and Rhizoctonia spp., biocontrol, 291

-Ralstonia solanacearum, oxidative environment, 773

-transcriptional responses, foliar and vascular, 245

Tomato spotted wilt virus, transcriptional responses, 1504

Tomlinson-Bulhot, L., 1203

Tooker, J. F., 551

Torrance, L., 381

Toruño, T. Y., 18

Tosmovirus spp., on tomato, silencing suppressor protein complementation, 1250

Trail, F., 1492

Trapphoff, T., 421

Trichoderma spp.

$-T$. atroviride, biocontrol gene, transporter membrane pump, 291

-T. harzianum, biocontrol agent, endopolygalacturonase, 1021

Triplett, E. W., 1624

Triplett, L. R., 1282

Trouvelot, S., 977

Tseng, Y.-H., 1379

Tsuge, S., 96

Tsuno, K., 96

Tudzynski, P., 987

Turina, M., 211

Turrà, D., 291

Tyler, H. L., 1624

Tytgat, T., 330

Udvardi, M., 800

Ugalde, R. A., 519

Ulrich, K., 1032

Umehara, Y., 259

Uribe, F., 703

Ustilago maydis, calcineurin, phosphorylation and antagonism, 1293 
Uyeda, I., 166

Vahling, C. M., 1011

Van Alfen, N. K., 211

van Arkel, G., 601, 630

van Brussel, A. A. N., 575

Van den Ackerveken, G., 1104

van den Broek, N. J. F., 1250

Van der Hoorn, R. A. L., 115

van der Lee, T., 1535

van der Vossen, E. A. G., 589, 601, 630

van der Zaal, B. J., 1356

van de Vondervoort, P. J. I., 1535

Van Eck, H. J., 601

van Esse, H. P., 245

van Gestel, K., 282

van Kregten, M., 1356

van Tuinen, D., 341

Varrelmann, M., 999

Venturi, V., 1514

Veronico, P., 1081

Verrier, J.-L., 868

Verticillium dahliae, on tomato, transcriptional responses, 245

Vesa, S., 868

Viana, C. J., 52

Vicedo, B., 1455

Vieweg, M. F., 63

Vigna unguiculata, nodulation, Sinorhizobium fredii, 575

Vinale, F., 291

Vinardell, J. M., 575, 1445

Vinatzer, B. A., 52

Virk, N., 1227

Visser, R. G. F., 601, 630, 1160, 1535

Vleeshouwers, V. G. A. A., 1535

Vogel, F., 655

von der Vossen, E. A. G., 1535

Vossen, J., 330

Waller, F., 1179

Walsh, J. A., 642

Walton, J. D., 1258, 1479

Wamboldt, Y., 1069

Wang, B., 411

Wang, G., 411
Wang, J., 1128

Wang, J.-X., 1143

Wang, X., 1128, 1227

Wang, Y., 498

Wang, Z., 235, 411

Warren, A., 52

Weckwerth, W., 1032, 1565

Wei, H.-Y., 1401

Wei, Y., 1601

Wendehenne, D., 977

Weng, F., 1601

Wheat

-Fusarium graminearum, tricothecene pathway, 899

-Fusarium head blight: deoxynivalenol, genes, 1588; transcriptome analysis, 1366; triaglyceride metabolism, 1492

- Heterodera avenae, class III peroxidase genes, 1081

- Mycosphaerella graminicola, NPP1 domain-containing protein, 790

-Pyrenophora tritici-repentis, hostselective toxin, 665

- silicon effect, transcriptomic analysis, stress, 1323

- Soilborne cereal mosaic virus, viral coat protein, leakiness, 560

-take-all, biocontrol, genes, 1611

Whisson, S. C., 630

White, C. E., 1116

Whitham, S. A., 123

Wielgus, S. M., 447

Wienkoop, S., 1565

Wilkinson, J. R., 1555

Williams, K. P., 1011

Williams, W. P., 1555

Wilson, R. A., 882

Wise, R. P., 311

Wolpert, T. J., 665

Wong, S. M., 1523

Woo, S. L., 291

Wright, W. D., 1258

Wu, A.-J., 391

Wu, J., 411

Wulff, B. B. H., 1191, 1203
Xanthomonas spp.

-X. campestris: hypersensitivity, Zur, 321; on cruciferous plants, type III effector genes, pathogenicity, 1401; on Solanaceae plants, type III effector protein, resistance, 655

-X. fuscans, on Phaseolus vulgaris: phyllosphere and transmission, 747

$-X$. oryzae, on rice, adhesion and virulence, 73; resistance and desaturase gene, 820 ; type III secretion effectors, 96

Xia, Y., 809

Xiao, Y., 964

Xu, R.-Q., 1401

Yajima, W., 783

Yamaji, Y., 677

Yan, S., 52

Yang, C., 123

Yang, S. L., 942

Yoshioka, H., 619

Yu, H., 1128

Yu, J.-J., 1143

Zaccardelli, M., 1577

Zerbini, F. M., 352

Zhang, C., 123

Zhang, H., 411, 1227

Zhang, N. W., 1160

Zhang, X., 411, 1143

Zhang, Y.-J., 1143

Zhang, Y.-N., 1143

Zhang, Z., 411

Zhao, Q., 411

Zhou, B., 411

Zhou, H., 176

Zhou, J.-M., 964

Zhou, L., 1011

Zhou, M.-G., 1143

Zhou, Z., 402

Zhu, Y., 498

Ziebell, H., 642

Ziegler, A., 381

Zou, J., 1601 